\title{
Behavioural problems in the first year after Severe traumatic brain injury: a prospective multicentre study
}

Clinical Rehabilitation 2017, Vol. 3I (4) 555-566 (C) The Author(s) 2016 Reprints and permissions: sagepub.co.uk/journalsPermissions.nav DOI: $10.1177 / 0269215516652184$ journals.sagepub.com/home/cre @SAGE

\author{
Catharina Nygren DeBoussard', Marianne Lannsjö ${ }^{2,3}$, \\ Maud Stenberg ${ }^{4}$, Britt-Marie Stålnacke ${ }^{4}$ and \\ Alison K Godbolt ${ }^{1,5}$
}

\begin{abstract}
Objective: To investigate the occurrence of behavioural problems in patients with severe traumatic brain injury during the first year after injury and potential associations with outcome. An additional post hoc objective was to analyse the frequency of behaviours with need for intervention from staff.

Design and setting: In a prospective population based cohort study II 4 patients with severe traumatic brain injury were assessed at three weeks, three months and one year after injury.

Main measures: Assessments included clinical examination and standardised instruments. Agitation was assessed with the Agitated Behaviour Scale, the course of recovery by the Rancho Los Amigo Scale and outcome by Glasgow Outcome Scale Extended.

Results: Agitation were most common at 3 weeks post injury and $28 \%(n=68)$ of the patients showed at least one agitated behaviour requiring intervention from staff. Presence of significant agitation at 3 weeks after injury was not associated with poor outcome. At 3 months agitation was present in II\% $(n=90)$ and apathy in 26 out of $8 \mathrm{I}$ assessed patients. At 3 months agitation and apathy were associated with poor outcome at one year.

Conclusions: Most agitated behaviours in the early phase are transient and are not associated with poor outcome. Agitation and apathy are uncommon at three months but when present are associated with poor outcome at one year after injury. In the early phase after a severe traumatic brain injury agitated behaviour in need of interventions from staff occur in a substantial proportion of patients.
\end{abstract}

\section{Keywords}

Severe traumatic brain injury, prognosis, agitation, behaviour, outcome, assessment

Received: 5 November 2014; accepted: 6 May 2016

'Department of Clinical Sciences, Karolinska Institutet, and University Department of Rehabilitation Medicine Stockholm, Danderyd Hospital, SE-I82 88 Stockholm, Sweden

${ }^{2}$ Sandviken Hospital and Uppsala University, Sweden ${ }^{3}$ Centre for Research and Development, Uppsala University/

County Council of Gävleborg Gävle, Sweden

${ }^{4}$ Department of Community Medicine and Rehabilitation,

Rehabilitation Medicine, Umeå University, Umeå, Sweden
${ }^{5}$ Formerly of Department of Rehabilitation Medicine,

University Hospital Uppsala and Uppsala University, Sweden

Corresponding author:

Catharina Nygren DeBoussard, Department of

Rehabilitation Medicine, Danderyd Hospital 18288

Stockholm, Sweden.

Email: catharina.nygren-deboussard@ds.se 


\section{Introduction}

Behavioural problems are one of many potential sequelae after a severe traumatic brain injury. Behavioural problems after traumatic brain injury may impact on outcome both indirectly i.e. on a patient's ability to make best use of any rehabilitation interventions offered and also directly ${ }^{1,2}$ i.e. on a patient's ability to live independently or work. Behaviour problems such as agitation may also affect the care-organisation's ability to provide care since these patients often have a period during their recovery with high personal supervision needs due to the different behaviours. ${ }^{3,4}$

The changes in behaviours that might occur after severe traumatic brain injury comprise both positive (e.g. agitation, disinhibition) and negative (e.g. apathy) symptoms. A variety of terms have been used to describe these behavioural changes..$^{5-9}$ Descriptions in the literature pertaining to TBI include variations of "neurobehavioral symptoms", "neuropsychatric consequences"6 as well as standard definitions of psychiatric disorders (e.g. according to Diagnostic System Manual $\mathrm{DSM}^{10}$ or International Classification of Disease ICD criteria), ${ }^{11}$ applied to patients after traumatic brain injury. The lack of a unified terminology might reflect overlap in symptomatology between different pathophysiological and diagnostic conceptual structures, despite these having diverging origins.

In the early period after a traumatic brain injury disorientation often occurs along with behavioural changes, most commonly agitation. The term post traumatic amnesia has therefore become established to describe the constellation of cognitive and behavioural changes that occur at this stage of recovery. ${ }^{12,13} \mathrm{~A}$ wide variety of figures $(11-70 \%)^{2}$ have been reported regarding the frequency of agitation during post traumatic amnesia. The timeframe for these early cognitive and behavioural changes varies from minutes to weeks after the traumatic brain injury. A somewhat separate body of literature considers behavioural changes later after injury ${ }^{14}$ and with a few exceptions ${ }^{15}$ studies have most often assessed behavioural changes at a single time point after injury, thus missing infor- mation on the time course of development and possible resolution of these symptoms.

The first aim of this prospective study was to explore rates of occurrence of behavioural problems in an unselected group of patients with severe traumatic brain injury at three time points during the first year after injury, and to test the hypothesis that the presence of behavioural problems, such as agitation and apathy early after injury is associated with outcome. An additional post hoc objective was to analyse the frequency of behaviours requiring need of intervention from staff to elucidate the need for appropriate staffing.

\section{Methods}

This study formed part of a prospective, multicentre, observational study of patients who had suffered severe traumatic brain injury (the "PROBRAIN" study). ${ }^{16-18}$

Study design has been described previously. ${ }^{16,17}$ In brief, inclusion criteria were severe, non-penetrating, traumatic brain injury requiring neurointensive care, with acute Glasgow Coma Scale score of $3-8,19$ in adult patients aged 18-65 years. Exclusion criteria were death or expected death within 3 weeks of injury.

A need for intensive care was an inclusion criterion as it is a marker of brain injury severity. In the study centres, patients are admitted to neurointensive care or managed in collaboration with a neurosurgeon if they require or are likely to require intracranial pressure monitoring, artificial ventilation or neurosurgery as part of brain injury management.

Patients were recruited prospectively by rehabilitation physicians from January 2010 until June 2011, with extended recruitment until December 2011 at two centres. The patient gave informed consent in cases where he/she had capacity. In the majority of cases the patient lacked capacity and the patient's nearest relative gave consent to inclusion. The study was reviewed by the regional ethical review board in Stockholm.

After inclusion, acute and socioeconomic data were obtained from medical records and via interview. Patients were then assessed prospectively, at three time points, three weeks (18-24 days), three months (75-105 days) and one year (350-420) days after injury. Patient characteristics are summarized 
Table I. Patient Characteristics $(n=|| 4)$.

Median age at injury
Median lowest Glasgow Coma Scale score* first 24
hours in patients not anesthetised
Cause of injury*

Median Length of stay - intensive care

Median duration of ventilation

Economic support at time of injury
Previous brain injury requiring hospitalisation Known drug or alcohol misuse at time of injury Gender

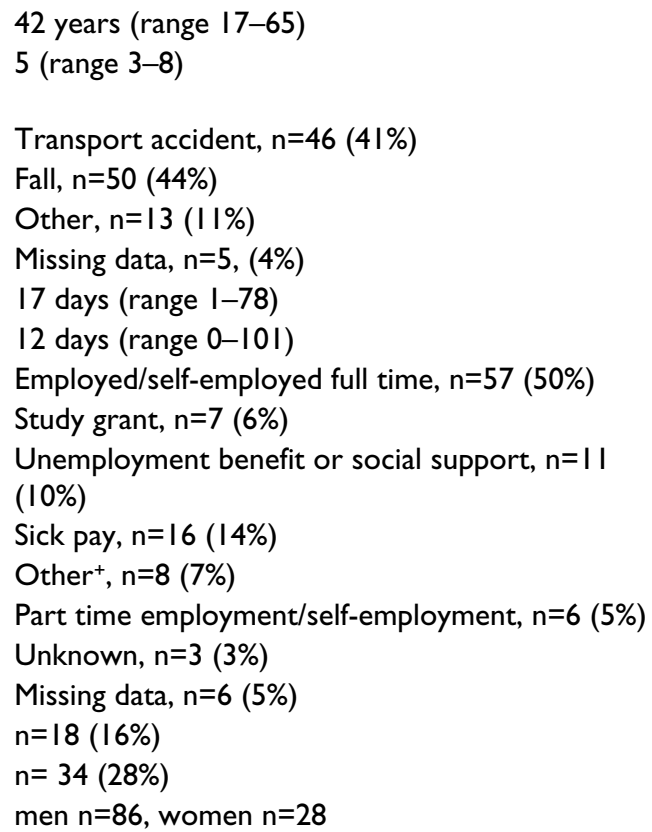

*Or derived Glasgow Coma Scale using conversion from Reaction Level Scale Score for patients exclusively assessed with the Reaction Level Scale $(n=42)$.

+"Other" includes parental pay, pension, other economic support, combinations of other categories.

in Table 1. Due to a minor protocol violation one patient was recruited shortly before their $18^{\text {th }}$ birthday.

Assessments included both clinical examination and a battery of standardised instruments. Based on clinical assessment at each of the three time points, the following clinical features were recorded as present, absent, or not assessable (for example due to ongoing anaesthesia or current medical complication): disorientation, disinhibition, apathy, anxiety and/or depression. In patients unable to communicate but not anesthetised, the presence of a possible Disorder of Consciousness was evaluated by clinical examination and with the Coma Recovery Scale Revised. ${ }^{20}$

Agitation was assessed with a Swedish translation of the Agitated Behavior Scale ${ }^{21}$ (supplementary Table), which is an established, structured, semi-quantitative assessment tool, designed for assessment of patients after TBI, and is based on direct clinical observation of the patient with rating of 14 different behaviours. The Agitated Behavior Scale has good reliability and validity. ${ }^{22}$ The Swedish translation was performed with permission of the scale authors, according to recommended procedures with forward and back translation. Agitated Behavior Scale total scores $>21$ represents clinically significant agitation, whilst item scores 2-4 are recorded when specific behaviours occur, with scores 3 or 4 for moderate and severe disturbances requiring redirection via intervention from staff.

Anxiety and/or depression were assessed with the Hospital Anxiety and Depression Scale (HADS), ${ }^{23}$ in cases where the patient had sufficient cognitive ability to understand the scale and answer. If necessary the questions were read to the patient who could answer verbally. The Hospital Anxiety and Depression Scale is an established screening tool for anxiety and depression. ${ }^{23}$ The 
Hospital Anxiety and Depression Scale has acceptable reliability, sensitivity and specificity in assessing symptom severity in anxiety and depression in different populations. ${ }^{24}$ Cut-offs for both subscales of 8 or higher were used to determine "caseness". 25

To allow consideration of our findings in relation to phase of recovery after severe traumatic brain injury, the Rancho Los Amigos Scale of cognitive functioning (RLAS) revised ${ }^{26}$ was assessed at each time point. The Rancho Los Amigos Scale of cognitive functioning is an established, descriptive scale, consisting of descriptions of different levels of functioning thought to reflect the recovery process. The Rancho Los Amigos Scale of cognitive functioning originally had 8 levels, while the revision added levels 9 and 10 to reflect the best levels of recovery. These levels have been grouped into three "blocks" representing broader stages of recovery.

Outcome at one year was measured using the Glasgow Outcome Scale Extended (GOSE). ${ }^{27}$ The Glasgow Outcome Scale Extended has good interrater reliability ${ }^{27}$ and validity, ${ }^{28}$ and is an established measure of global outcome after traumatic brain injury. A standardised interview ${ }^{27}$ was used to support good inter-rater reliability. For some analyses it was necessary to dichotomise the Glasgow Outcome Scale Extended findings into "good" and "poor" outcome. This division was made in accordance with previous definitions ${ }^{29}$ of "good" and "poor" outcome: For those alive at one year, Glasgow Outcome Scale Extended 2-4 was considered a "poor" outcome, and Glasgow Outcome Scale Extended 5-8 a "good" outcome. As our focus was on evaluation of the possible impact of behavioural dysfunctions on the patients' ability to participate in rehabilitation and long term outcome rehabilitation, it was not meaningful to include patients who did not receive rehabilitation because they had died (i.e. Glasgow Outcome Scale Extended 1), in these analyses.

An externally validated acute prognostic model the CRASH acute prognostic model - was used to control for acute injury severity. We used the online calculator for the CRASH prognostic model (available at http://crash2.1shtm.ac.uk/Risk\%20calculator/ index.html) to calculate the risk of an unfavourable outcome at 6 months. The model is based on 10 acute prognostic variables; age, pupil reaction, acute GCS score, country, presence or absence of 5 specified acute computed tomographic brain findings and the outcome calculated is equivalent to dichotomised Glasgow Outcome Scale Extended.

\section{Statistical analysis}

Analysis was performed with SPSS version 22. Non-parametric tests were used to test significance of associations with outcome (dichotomised Glasgow Outcome Scale Extended) due to skewed data. Mann-Whitney test was used for continuous variables. For tests of relative frequencies, Chisquared tests were used where the number of observations in each cell was 5 or greater and Fisher's exact test when this condition was not met. Statistical significance was set at $p<0.05$. Choice of analyses was informed by the hypotheses and by the need to minimize the risk of false positive results due to multiple comparisons.

\section{Results}

The severity of brain injury in these patients is reflected by the need for intensive care and neurosurgical interventions: 110 patients $(96 \%)$ required artifical ventilation. $100(88 \%)$ required monitoring of intracranial pressure. 52 (46\%) underwent surgical evacuation of a traumatic intracerebral bleed (subdural, extradural or intracerebral) and $41(36 \%)$ underwent a procedure to correct cerebrospinal fluid dynamics (ventriculostomy or shunt insertion).

Patients who withdrew were similar to those who continued in terms of median age (30 compared to 42 years, Mann-Whitney test $p=0.24$ ) and median acute Glasgow Coma Scale score or reaction level scale-derived Glasgow Coma Scale score ( 5 in both groups, Mann-Whitney test $p=0.55$ ).

Table 2 shows a description of conscious state according to CRS-R, clinical behavioural examinations and results of the Agitated Behaviour Scale and Hospital Anxiety and Depression Scale available at each time-point.

\section{Agitation}

The Agitated Behaviour Scale was administered for patients who were conscious or minimally 
Table 2. Behavioural problems and emotional disturbances during the first year after a severe traumatic brain injury.

\begin{tabular}{|c|c|c|c|c|}
\hline & & 3 weeks & 3 months & I year \\
\hline \multirow[t]{3}{*}{ Follow up status } & Continued in study & III & 105 & 100 \\
\hline & dead & I & 5 & 7 \\
\hline & withdrawn & 2 & 4 & 7 \\
\hline \multirow[t]{6}{*}{ Conscious state } & anesthetised & 12 & 0 & 0 \\
\hline & coma & 6 & 0 & 0 \\
\hline & vegetative state & 19 & 10 & 5 \\
\hline & minimally conscious state & 13 & II & 5 \\
\hline & fully conscious & 58 & 84 & 90 \\
\hline & Missing data & 3 & 0 & 0 \\
\hline $\begin{array}{l}\text { Agitated Behaviour Scale (assessed in } \\
\text { patients fully conscious or minimally }\end{array}$ & $\begin{array}{l}\text { Number above cut off }>21 \text { points } \\
\text { (n assessed) }\end{array}$ & $8(68)$ & $10(90)$ & $6(87)$ \\
\hline conscious) & Missing data & 3 & 5 & 8 \\
\hline $\begin{array}{l}\text { Apathy (clinical assessment in patients } \\
\text { fully conscious) }\end{array}$ & $\begin{array}{l}\text { Present ( } \mathrm{n} \text { assessed) } \\
\text { Missing data }\end{array}$ & $\begin{array}{l}18(51) \\
7\end{array}$ & $\begin{array}{c}26(8 I) \\
3\end{array}$ & $\begin{array}{l}31(84) \\
6\end{array}$ \\
\hline $\begin{array}{l}\text { Disinhibition (clinical assessment in } \\
\text { patients fully conscious) }\end{array}$ & $\begin{array}{l}\text { Present ( } \mathrm{n} \text { assessed) } \\
\text { Missing data }\end{array}$ & $\begin{array}{l}6(50) \\
8\end{array}$ & $\begin{array}{l}\text { II (80) } \\
\quad 4\end{array}$ & $\begin{array}{l}17(85) \\
5\end{array}$ \\
\hline \multirow[t]{4}{*}{$\begin{array}{l}\text { Disorientation (clinical assessment in } \\
\text { patients fully conscious) }\end{array}$} & $\begin{array}{l}\text { Present ( } \mathrm{n} \text { assessed) } \\
\text { Not assessable } \\
\text {-due to brain injury related }\end{array}$ & $2 I(53)$ & $19(78)$ & $15(85)$ \\
\hline & communication problems & 3 & 4 & 0 \\
\hline & -due to tracheostomy & 1 & 0 & $\mathrm{I}$ \\
\hline & Missing data & 1 & 2 & 4 \\
\hline \multirow[t]{8}{*}{ Hospital Anxiety and Depression Scale } & $\begin{array}{l}\text { subscale depression number above } \\
\text { cut off }>7 \text { ( } \mathrm{n} \text { assessed) }\end{array}$ & $7(36)$ & $\mathrm{II}(75)$ & $15(76)$ \\
\hline & $\begin{array}{l}\text { subscale anxiety number above cut } \\
\text { off }>7 \text { ( } n \text { assessed) }\end{array}$ & $10(36)$ & $16(75)$ & $16(76)$ \\
\hline & Not assessable & & & \\
\hline & $\begin{array}{l}\text {-low conscious state } \\
\text {-brain injury related } \\
\text { communication problems (aphasia }\end{array}$ & 50 & 21 & 10 \\
\hline & or incomprehensible speech) & 9 & 6 & 2 \\
\hline & -tracheostomy & 4 & 0 & 0 \\
\hline & -on going medical complications & 10 & 3 & 0 \\
\hline & $\begin{array}{l}\text {-Hospital Anxiety and Depression } \\
\text { Scale not completed due to } \\
\text { logistical reasons }\end{array}$ & 2 & 0 & 12 \\
\hline
\end{tabular}

conscious. Data were missing for $3 / 71$ patients assessable at 3 weeks, 5/95 patients at 3 months, and $8 / 95$ patients at one year, 57 patients had complete data on Agitated Behaviour Scale at all time points. Commonest agitated behaviours, as assessed with Agitated Behavioural Scale were "short attention span, easy distractibility, inability to concentrate" and "impulsivity" at all study time points (Figure 1). Of the 57 patients with data on Agitated Behavioural Scale at all time points none were agitated at all time points (Figure 2). Subacute medical complications were common in this cohort ${ }^{30}$ being present in two thirds of patients at the time of 3 weeks follow up. Four of the eight patients displaying agitation at 3 week had a medical complication (three infections, one new fracture).

According to the Agitated Behaviour Scale, item scores 3 or 4 for moderate and severe disturbances represent behaviours that require redirection via intervention from staff. At three weeks, 19 patients out of 68 assessed had behaviours requiring redirection via intervention from staff, the corresponding figure at three months was 15 out of 90 assessed and at 1 year 12 patients out of 87 assessed. 


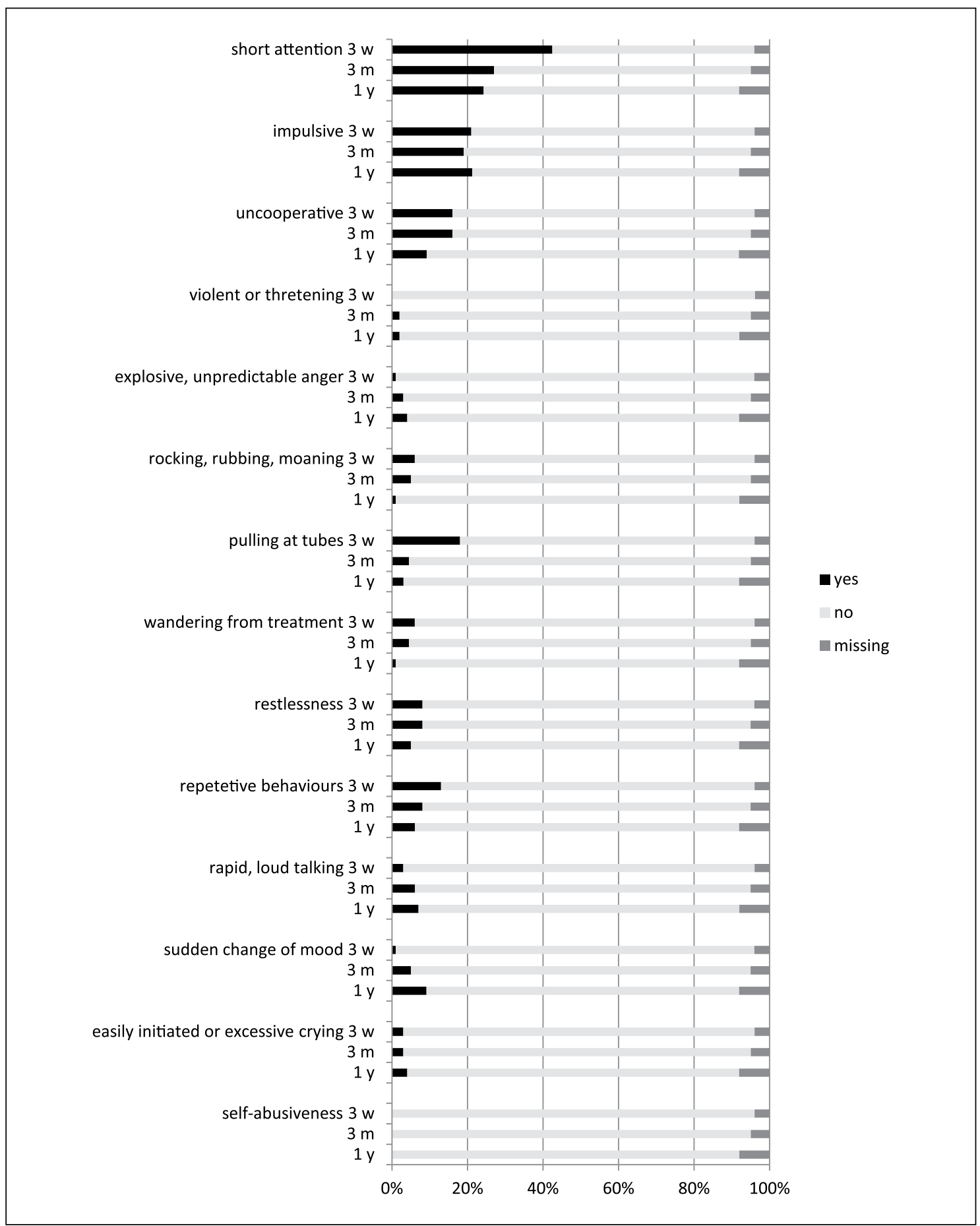

Figure I. Percentage of patients with or without the specified behaviour according to the Agitated Behavioural Scale at 3 weeks, 3 months or one year after brain injury. 
Agitated behaviours occurred even in patients who were oriented. At 3 weeks after injury of the 32 patients with $\geqslant 1$ agitated behaviour 7 were oriented, at 3 months after injury 14 out of 35 patients and at 1 year after injury 16 out of 28 patients.

During the middle stages of recovery, as described by Rancho Los Amigo Scale levels 4-6, agitation is considered a typical although a temporary phase in the pathway to recovery. Table 3 illustrates the number of patients with $\geqslant 1$ agitated behaviour at the different time points and the stage of recovery according to Rancho Los Amigo Scale.

Disinhibition was evaluated by clinical examination in fully conscious patients. Disinhibition was uncommon at 3 weeks ( 6 patients out of 50 assessable), at three months $14 \%$ of the assessable patients $(n=80)$ and at 1 year $20 \%$ of the patients $(n=85)$, Table 2.

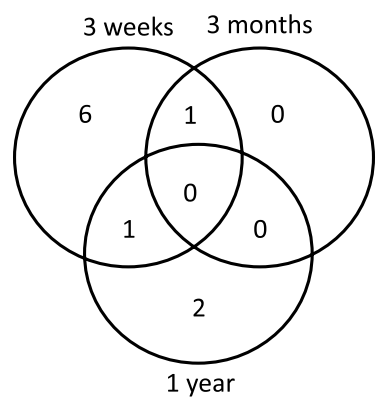

Figure 2. Number of agitated patients at each study time point (Agitated Behavioural Scale score >21), $n=57$. Patients with complete longitudinal data.
Apathy. The proportion of patients with apathy evaluated by clinical examination in patients fully conscious did not change during the first year, at three weeks $35 \%(n=51)$, at 3 months $32 \%(n=81)$ and at 1 year $37 \%(n=84)$, table 2 .

\section{Association of behavioural problems, anxiety and depression with outcome}

The presence of significant agitation, according to the Agitated Behaviour Scale, at 3 weeks after injury was not associated with poor outcome (dichotomised Glasgow Outcome Scale Extended, chi-squared $p=0.60)$. Neither was there an association between significant agitation at 3 weeks and independent living at one year (6/10 agitated at 3 weeks and 39/54 patients not agitated at 3 weeks were living independently at one year, chisquared, not significant, $p=0.44)$. However the presence of clinically significant agitated behaviour at 3 months was associated with poor outcome: $5 / 6$ patients with clinically significant agitated behaviour and 20/79 patients without clinically significant agitated behaviour had a poor outcome at one year (Fisher's exact test, $p=0.01)$.

Apathy at 3 weeks was not associated with outcome, whilst apathy at 3 months was associated with poor outcome at one year $(11 / 25$ with apathy and $5 / 52$ without apathy at 3 months had a poor outcome at one year, $p=0.002$, Fisher's exact test).

It was possible to administer the Hospital Anxiety and Depression Scale in about a third of patients (36/109), at 3 weeks after injury, Table 2.

Table 3. Presence of agitated behaviour during the recovery phases according to Rancho Los Amigo Scale Revised.

Rancho Los Amigo Scale R

Patients with more than one agitated behaviour

\begin{tabular}{lccc}
\hline & 3 weeks & 3 months & I year \\
Block I, level I-3 in Rancho los Amigo Scale R & 9 & 3 & 0 \\
Block 2, level 4-6 in Rancho Los Amigo Scale R & 11 & 11 & 8 \\
\hline Block 3, level 7-10 in Rancho Los Amigo Scale R & 12 & 19 & 24 \\
\hline
\end{tabular}


Table 4. Associations between behavioural problems and outcome one year after severe TBI.

\begin{tabular}{|c|c|c|c|c|}
\hline Feature & $\begin{array}{l}\text { Time point } \\
\text { after injury for } \\
\text { assessment }\end{array}$ & $\begin{array}{l}\text { Significantly associated } \\
\text { with poor outcome } \\
\text { (dichotomised Glasgow } \\
\text { Outcome Scale) }\end{array}$ & $p$ value & Test* \\
\hline $\begin{array}{l}\text { Acute injury severity (acute } \\
\text { Glasgow Coma Scale) }\end{array}$ & On admission & No & 0.19 & Chi-squared \\
\hline \multirow{2}{*}{$\begin{array}{l}\text { Clinically significant } \\
\text { agitation }\end{array}$} & 3 weeks & No & 0.60 & Chi-squared \\
\hline & 3 months & Yes & 0.01 & Fisher's exact test \\
\hline \multirow[t]{2}{*}{ Apathy } & 3 weeks & No & 0.34 & Fisher's exact test \\
\hline & 3 months & Yes & 0.002 & Fisher's exact test \\
\hline
\end{tabular}

*Fisher's exact test used when any cell had $n<5$.

Communicative ability, level of consciousness, or medical complications in the remainder made this impossible.

Depression and apathy at 3 months were not significantly associated. 18/21 apathetic patients scored below cut-off on the Hospital Anxiety and Depression Scale depression subscale, and 8/11 depressed patients were not considered apathetic (Fisher's exact test, $p=1.0$ ). Associations of agitation and apathy with outcome at one year are summarized in Table 4.

There was also a negative association between agitation at 3 months and independence at one year i.e. Glasgow Outcome Scale Extended score $>4$. $0 / 7$ patients agitated at 3 months and 54/80 patients not agitated at 3 months were living independently at one year ( $p=0.001$, Fisher's Exact Test). This was not explained by acute injury severity (no difference in acute injury severity as assessed by the CRASH composite, ${ }^{29}$ between patients agitated [median $67 \%$ risk of poor outcome, interquartile range (IQR) $53-89 \%$ ] and not agitated [median $73 \%$ risk of poor outcome, interquartile range (IQR) $56-88 \%$ ] at 3 months).

It was hypothesised that patients who were agitated $(\mathrm{ABS}>21)$ may have a longer wait before rehabilitation admission after discharge from intensive care. There was however no difference in the number of days between intensive care for patients with agitation at 3 weeks (median 12.5 days, interquartile range [IQR] 2.5-38) compared to those without agitation (median 11 days, IQR 0-18), Mann-Whitney $p=0.33$.

\section{Discussion}

This prospective study demonstrates a broad span of behavioural problems including positive symptoms (agitation) and negative symptoms (apathy) during the first year after a severe traumatic brain injury. These behaviours were most common early after the injury. The presence of significant agitation, according to the Agitated Behaviour Scale, at 3 weeks after injury was not associated with poor outcome. This is in line with the assumption that agitation reflects a temporary phase in the pathway to recovery. However, agitation at 3 months was associated with poor outcome at one year. Thus, agitation at this stage deserves attention and may be a target for interventions in order to improve long-term outcome after severe TBI.

A strength of this study is the prospective, unselected multicentre recruitment, independent of rehabilitation admission, and high follow-up rates, which guard against selection bias. Many previous studies ${ }^{31,32}$ of behavioural dysfunctions are subject to potential selection bias e.g. due to recruitment, only of patients admitted to inpatient rehabilitation.

Behavioural problems in general, and agitation in particular, need to be considered within the context of stage of recovery. There was a dissociation between the presence of agitation and stage of recovery as rated with the Rancho Los Amigo scale in our study, such that agitation could not simply be explained by different trajectories of recovery. Furthermore, we found an incomplete association 
between agitation and disorientation, suggesting that conceptualising agitation solely as something that occurs during post traumatic amnesia is too simplistic. A previous retrospective Australian study $^{33}$ found that agitation continued after emergence from post-traumatic amnesia in $27 \%$ of patients, adding some support to this. For patients not agitated at the early assessment, late emergence of agitation rated as globally significant was uncommon.

Agitation can be a significant practical problem when caring for patients after traumatic brain injury and its management very resource intensive. E.g., $32 \%$ of agitated patients in one study ${ }^{2}$ required constant supervision, which very few units are funded for. The proportion of patients with clinically significant agitation in our study, (according to the cut-off scores defined by the scale authors), was somewhat lower than expected, at $12 \%$ or less at all time points. One possible explanation is related to the design of our study, with assessment standardised according to time after injury, with the first assessment at 3 weeks (18-24 days). Although standardising assessment to certain timepoints after the injury enabled valid comparisons in other respects, we may have missed patients who had already passed through an agitated period as well as those who were yet to become agitated (particularly for patients who were anesthetised or were in coma or vegetative state at 3 weeks). This must also be considered when interpreting the observed trend towards patients with more severe injuries having more agitated behaviours at 3 weeks. Thus, even though it seems reasonable that more severely injured patients develop agitation more often, less severely injured patients may already have passed through an agitated period before the 3 week assessment.

Agitated Behaviour Scale item scores, each of which is related to the need for intervention from staff, may in some respects be a more accurate measure of care burden and resource needs than the global Agitated Behaviour Scale assessment. If any one of these specific behaviours occurs to a degree requiring intervention from staff, then staffing levels must be sufficient to allow such intervention. For example, a quarter of patients required intervention from staff due to "Short attention span, easy distractibility, inability to concentrate" during the 3 week assessment. In contrast, certain items on the Agitated Behaviour Scale detected no patients who needed intervention from staff, which is reassuring in terms of patient and staff safety (e.g. "violent and or threatening violence toward people or property" and "sudden changes of mood").

Data from prospective, long-term studies in this area are scarce. A previous retrospective study ${ }^{33}$ used a case note review to assess rates of agitation during patients' entire inpatient rehabilitation stay after traumatic brain injury, ${ }^{33}$ with data on agitation during acute care available for a subset. $41 \%$ of these patients had severe injury. The authors found that $70 \%$ of patients were agitated at some point during their inpatient stay, with onset of agitation a mean (SD) of 6.7 (9.9) days (range 0-41 days) after injury, and duration of agitation a mean (SD) of 23.9 (20.9) days. If our cohort had similar time frames for the onset and duration of agitation, then most cases would be expected to be captured by assessment at 3 weeks. However, differences in study design (retrospective/prospective) and injury severity (more severe in our cohort) limit comparisons.

Our observation that presence of agitated behaviours at 3 months was a strong predictor of agitated behaviour and dependence on others at one year may be considered not only with regard to future intervention studies but also with regard to current clinical practice. The likelihood of resolution of agitation is an important factor when planning continuing care. This observation is also in accordance with a recent study by Singh et al. ${ }^{31}$ They found that over a third of patients with TBI admitted to a rehabilitation unit manifested agitation, and that duration of agitation was associated with poor outcome according to the Glasgow Outcome Scale Extended. ${ }^{31}$

In this report, we took an inclusive approach, in order to capture as many behaviours as possible that could be interpreted as agitation. For this reason we included patients in the minimally conscious state (MCS) in the analyses of agitation. By definition, ${ }^{20}$ 
these patients lack functional communication ability and the ability to use objects functionally. However at the higher levels of minimally conscious state patients may show behaviours that could be interpreted as agitation, for example pulling at tubes, resisting care, vocalising. We acknowledge that the application of the concept of agitation to this patient group is not a matter of consensus, and it may be argued that these patients should be excluded when assessing rates of agitation. Excluding these patients from the analyses, would give slightly higher percentage rates for agitation at each time point.

Although apathy at 3 months was uncommon, there was a strong association with poor outcome that was not explained by depression, indicating that studies of specific treatment for apathy may be justified. It should be pointed out, that symptoms of apathy overlap with symptoms of depression and that five of the nine features of depression described in Diagnostic System Manual-IV, (significant weight loss, insomnia, psychomotor agitation or retardation, fatigue or loss of energy, diminished ability to think or concentrate) are also common features in the post-acute period after severe traumatic brain injury, independently of any depression. The presence of five symptoms is also sufficient to fulfil criteria for a diagnosis of depression according to Diagnostic System Manual -IV, if accompanied by reduced interest in activities, which could be due to apathy in patients who have suffered severe traumatic brain injury. The risk of inappropriate diagnosis of depression is thus considerable. The ability of patients to self-report symptoms varies, and the use of existing standardised rating scales developed for use in the general population may not be appropriate. ${ }^{34}$ Thus, development of valid criteria for behavioural problems and for post-injury mood disturbances would be one priority in future research. A better understanding of relations between behavioural problems, ratings of low mood, and response to rehabilitation interventions, is needed before progressing to studies of the effectiveness of treatment directed at mood disturbance on medium and long term outcome.

Weekly contact with intensive care units guarded against selection bias, but we cannot exclude that some patients were admitted and discharged between contacts, and would therefore have been missed from the recruitment process. Some data was also missing. To some extent this was unavoidable as data collection was part of a wide ranging follow up study with many assessment instruments, and the necessity of assessing patients over wide geographical areas, which meant that on some occasions not all study assessments could be completed in the available time.

This study focuses on an age-defined subset of patients with severe traumatic brain injury aged 18-65 years, for the following reasons: Patients below 18 years of age receive rehabilitation within paediatric services which are separately organised in Sweden and it was not logistically possible to include them. Exclusion of older patients avoided confounders such as the impact of co-morbidities (the likelihood of which rises with age), different life situations and expectations regarding behavioural and emotional demands, in elderly patients.

The Agitated Behaviour Scale was chosen to assess agitation as it is a standardized, valid and reliable instrument that was suitable for use at all study time points. However it only captures behaviours that occur during the assessment. Infrequent but potentially troublesome behaviours may thus not be captured.

The exploratory nature of this study necessitated the use of multiple comparisons, with some risk of type 1 error, and confirmatory studies are advised.

\section{Clinical messages}

- Both agitation and apathy are associated with poor outcome.

- Behavioural problems during the first year after severe traumatic brain injury are dynamic, complex and incompletely captured by existing diagnostic frameworks.

\section{Acknowledgements}

We thank the patients and their relatives, the PROBRAIN collaborators*, the clinical staff of our units, and our 
neurosurgical colleagues for allowing recruitment of their patients. We also thank Dr Anna Tölli, Danderyd Hospital and Karolinska Institute, Dr Kristina Lindgren, Karlstad Hospital, Dr Björn Johansson, University Hospital Uppsala and Dr Christer Tengvar, University Hospital Uppsala, who assisted in assessment of patients. Paramedical staff contributing to the study included Stina Gunnarsson and Marina Byström Odhe, (Linköping), Anna-Lisa Nilsson (Umeå), Maria Sandgren (Stockholm), Staffan Stenson (Uppsala), and Siv Svensson (Gothenburg). Seija Lund was nurse coordinator in Stockholm and Ingrid Morberg in Gothenburg. Lisbet Broman gave advice on statistical aspects. Professor Rickard Levi organised financial support in the northern region of Sweden.

*PROBRAIN collaborators: the authors together with Dr Marie Lindgren, MD, Department of Clinical Rehabilitation Medicine, County Council, Linköping, Sweden, Dr Trandur Ulfarsson, MD, PhD, Dept of Rehabilitation Medicine, Sahlgrenska University Hospital, Gothenburg, Sweden, Prof Rickard Levi Rehabilitation Medicine, Umeå University, Umeå, Sweden and Dr Gudrun Karlsdottir, Dept of Rehabilitation Medicine, Landspitalinn, University Hospital, Reykjavik, Iceland.

\section{Declaration of Conflicting Interests}

The author(s) declared no potential conflicts of interest with respect to the research, authorship, and/or publication of this article.

\section{Funding}

The author(s) disclosed receipt of the following financial support for the research, authorship, and/or publication of this article: This study was supported by grant 060833 and 130095 from AFA insurance. AKG has received support from ALF-grants from Uppsala University Hospital and Danderyd Hospital. ML has received ALF-grants from Regional Research Council in the Region of Uppsala-Örebro, MS has received support from ALF-grants from Umeå University and Umeå University Hospital.

\section{References}

1. Bogner JA, Corrigan JD, Fugate L, et al. Role of agitation in prediction of outcomes after traumatic brain injury. Am J Phys Med Rehabil 2001; 80: 636-644.

2. McNett M, Sarver W and Wilczewski P.The prevalence, treatment and outcomes of agitation among patients with brain injury admitted to acute care units. Brain Inj 2012; 26: 1155-1162.
3. Bailey M, Amato S and Mouhlas C.A creative alternative for providing constant observation on an acute-braininjury unit. Rehabil Nurs 2009; 34: 11-6, 23.

4. Becker C.Nursing care of the brain injury patient on a locked neurobehavioral unit. Rehabil Nurs 2012; 37: 171-175.

5. Arango-Lasprilla JC, Ketchum JM, Drew A, et al. Neurobehavioural symptoms 1 year after traumatic brain injury: a preliminary study of the relationship between race/ethnicity and symptoms. Brain Inj 2012; 26: 814-824.

6. Bhalerao SU, Geurtjens C, Thomas GR, et al. Understanding the neuropsychiatric consequences associated with significant traumatic brain injury. Brain Inj 2013; 27: 767-774.

7. Lippert-Gruner M, Kuchta J, Mellmich M, et al. Neurobehavioural deficits after severe traumatic brain injury (TBI). Brain Inj 2006; 20: 569-574.

8. McAllister TW.Neuropsychiatric sequelae of head injuries. Psychiatr Clin North Am 1992; 15: 395-413.

9. Riggio $\mathrm{S}$ and Wong M.Neurobehavioral sequelae of traumatic brain injury. Mt Sinai J Med 2009; 76: 163-172.

10. Diagnostic and Statistical Manual of Mental Disorders. V ed. 2013, Arlington, Virginia: American Psychiatric Publishing.

11. International Classification of Diseases, Tenth Revision (ICD-10). Atlanta (GA): National Center for Health Statistics. Available at: http://www.cdc.gov/nchs/icd/ icd10.htm.

12. Marshman LA, Jakabek D, Hennessy M, et al. Posttraumatic amnesia. J Clin Neurosci 2013; 20: 1475-1481.

13. Nakase-Thompson R, Sherer M, Yablon SA, et al. Acute confusion following traumatic brain injury. Brain Inj 2004; 18: 131-142.

14. Worthington A, Matthews S, Melia Y, et al. Cost-benefits associated with social outcome from neurobehavioural reahabilitation. Brain Inj 2006; 20: 947-957.

15. Bombardier CH, Fann JR, Temkin NR, et al. Rates of major depressive disorder and clinical outcomes following traumatic brain injury. JAMA 2010; 303: 1938-1945.

16. Godbolt $\mathrm{AK}$, Deboussard $\mathrm{CN}$, Stenberg $\mathrm{M}$, et al. Disorders of consciousness after severe traumatic brain injury: a Swedish-Icelandic study of incidence, outcomes and implications for optimizing care pathways. $J$ Rehabil Med 2013; 45: 741-748.

17. Godbolt AK, Stenberg M, Lindgren $M$, et al. Associations between care pathways and outcome 1 year after severe traumatic brain injury. J Head Trauma Rehabil 2015; 30: E41-E51.

18. Stenberg M, Godbolt AK, Nygren De Boussard C, et al. Cognitive Impairment after Severe Traumatic Brain Injury, Clinical Course and Impact on Outcome: A Swedish-Icelandic Study. Behav Neurol 2015: Epub ahead of print 9 December 2015 .

19. Teasdale $\mathrm{G}$ and Jennett B.Assessment of coma and impaired consciousness. A practical scale. Lancet 1974; 2: 81-84. 
20. Giacino JT, Kalmar K and Whyte J.The JFK Coma Recovery Scale-Revised: measurement characteristics and diagnostic utility. Arch Phys Med Rehabil 2004; 85: 2020-2029.

21. Corrigan JD.Development of a scale for assessment of agitation following traumatic brain injury. J Clin Exp Neuropsychol 1989; 11: 261-277.

22. Bogner JA, Corrigan JD, Stange M, et al. Reliability of the Agitated Behavior Scale. J Head Trauma Rehabil 1999; 14: 91-96.

23. Zigmond AS and Snaith RP.The hospital anxiety and depression scale. Acta Psychiatr Scand 1983; 67: 361-370.

24. Schwarzbold ML, Diaz AP, Nunes JC, et al. Validity and screening properties of three depression rating scales in a prospective sample of patients with severe traumatic brain injury. Rev Bras Psiquiatr 2014; 36: 206-212.

25. Hung CI, Liu CY, Wang SJ, et al. The cut-off points of the Depression and Somatic Symptoms Scale and the Hospital Anxiety and Depression Scale in detecting nonfull remission and a current major depressive episode. Int J Psychiatry Clin Pract 2012; 16: 33-40.

26. Hagen C. Rancho Los Amigo Scale of cognitive functioning. Available at: http://www.tbims.org/combi/lcfs/ index.html

27. Wilson JT, Pettigrew LE and Teasdale GM.Structured interviews for the Glasgow Outcome Scale and the extended Glasgow Outcome Scale: guidelines for their use. J Neurotrauma. 1998; 15: 573-585.

28. Levin HS, Boake C, Song J, et al. Validity and sensitivity to change of the extended Glasgow Outcome Scale in mild to moderate traumatic brain injury. J Neurotrauma 2001 ; 18: 575-584.

29. Perel P, Arango M, Clayton T, et al. Predicting outcome after traumatic brain injury:practical prognostic models based on large cohort of international patients. BMJ 2008; 336: 425-429.

30. Godbolt AK, Stenberg M, Jakobsson J, et al. Subacute complications during recovery from severe traumatic brain injury: frequency and associations with outcome. BMJ Open 2015; 5: e007208.

31. Singh R, Venkateshwara G, Nair KP, et al. Agitation after traumatic brain injury and predictors of outcome. Brain Inj 2014; 28: 336-340.

32. Whelan-Goodinson R, Ponsford $\mathrm{J}$ and Schonberger M.Association between psychiatric state and outcome following traumatic brain injury. J Rehabil Med 2008; 40: 850-857.

33. Nott MT, Chapparo C and Baguley IJ.Agitation following traumatic brain injury: an Australian sample. Brain Inj 2006; 20: 1175-1182.

34. Seel RT, Macciocchi S and Kreutzer JS.Clinical considerations for the diagnosis of major depression after moderate to severe TBI. J Head Trauma Rehabil 2010; 25: 99-112. 


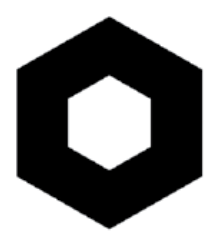

\title{
British Society of
} Rehabilitation Medicine

Promoting quality through

education and standards

\section{Forthcoming events ...}

\author{
Specialist Rehabilitation Medicine Course \\ 18 \& 19 May 2017 - Derby \\ $3^{\text {rd }}$ Sheffield Spinal Cord Injury Course \\ 5-7 July 2017 - Sheffield
}

BSRM 2017 Annual Scientific Meeting - Cambridge

More details coming soon

BSRM is also collaborating with two international events in 2017

$14^{\text {th }}$ Congress of the European Federation for Research in Rehabilitation 2017

24-27 May 2017 - Glasgow

www.efrr2017.com/

$\&$

Rehab Week 2017

17-20 July 2017 - London

www.rehabweek.org/2017

Regular updates including abstract submission and registration at www.bsrm.org.uk

The British Society of Rehabilitation Medicine is the Society which represents the specialty of Rehabilitation Medicine. It promotes an understanding of the specialty through the education and development of clinical guidelines and standards

BSRM, c/o Royal College of Physicians, 11 St Andrews Place,

London NW1 4LE (tel: +44(0)1992 638865) Www.bsrm.org.uk

Registered Charity Number 293196 
